\title{
CIENCIAS BÁSICAS EN URUGUAY: EL PEDECIBA, 33 AÑOS DESPUÉS
}

\author{
Adriana Chiancone \\ Doctora -PhS- en Estudios Sociales de la \\ Ciencia y la Tecnología \\ Universidad CLAEH (Uruguay) \\ Contacto: achiancouniversidad@gmail.com \\ ORCID: 0000-0002-4332-3702
}

Recibido: 14 de julio de 2019

Aceptado: 12 de agosto de 2019

DOI: $10.25087 /$ resur8a3

\section{Resumen}

El Programa de Desarrollo de Ciencias Básicas (PEDECIBA), es un programa de desarrollo científico, que surgió en Uruguay en un contexto de transición política, de un gobierno militar a una democracia. Se inició a fines de 1986, en las áreas de biología, física, informática, matemática y química, implementado por la Universidad de la República y el Ministerio de Educación y Cultura. Treinta y tres años después de su fundación, en este artículo se intenta identificar algunos cambios y formas de articulación del programa en el actual contexto institucional nacional.

El PEDECIBA estuvo caracterizado desde sus orígenes por disponer de una mínima estructura y por una gran flexibilidad. Es una red de investigadores administrada por los propios investigadores, a la que se han integrado nuevos campos interdisciplinarios y se ha ampliado el número de instituciones uruguayas donde trabajan los científicos del programa. Con algunos desafíos asociados a los cambios científicos a nivel global, e institucionales y de políticas locales, el programa mantiene su importancia en la formación avanzada de recursos humanos y en la coordinación y creación de un espacio de reunión de investigadores.

Palabras clave: PEDECIBA, ciencias básicas, posgrados científicos, disciplinas, institucionalización. 


\begin{abstract}
The Programme for the Development of Basic Sciences (PEDECIBA) was created in Uruguay as a scientific development program within a context of political transition. The program started at the end of 1986 in the areas of Biology, Physics, Computer Science, Mathematics and Chemistry, implemented by the University of the Republic (UDELAR) and the Ministry of Education and Culture (MEC). Thirtythree years after its foundation, this article aims to identify some transformations and new forms of program articulation within the current institutional national context.

PEDECIBA has been characterized since its origins by a flexible organization, without bureaucratic structures. It is a researcher's network, managed by the program's scientists. New interdisciplinary fields have been integrated to PEDECIBA and scientists do work in a larger number of Uruguayan institutions created in these 33 years.

The program faces some challenges related to global scientific transformations and local institutional and political changes. Nevertheless, PEDECIBA maintains its importance not only in postgraduate training but also in the coordination and creation of a space for researchers.
\end{abstract}

Key words: PEDECIBA, Basic Sciences, Post Degree Courses, Disciplines, Institutionalization.

\title{
1. Introducción
}

El Programa de Desarrollo de Ciencias Básicas (PEDECIBA), nació como un programa de desarrollo científico, ejecutado por el gobierno de Uruguay con la ayuda del Programa de Naciones Unidas para el Desarrollo (PNUD) y la Organización de Naciones Unidas para la Educación, la Ciencia y la Cultura (UNESCO), que se inició a fines de 1986, en las áreas de biología, física, informática, matemática y química, ejecutado por la Universidad de la República (UDELAR) y el Ministerio de Educación y Cultura (MEC).

El PEDECIBA representó entre otras cosas, una iniciativa para fortalecer la comunidad científica uruguaya. Este propósito implicaba básicamente la recuperación de los recursos humanos que se encontraban fuera del país, la creación de cargos "full-time", mejorar la infraestructura de laboratorios y bibliotecas, y la posibilidad de que existieran becas para realizar estudios de posgrado. Esta experiencia aprovechó ciertas características de la difícil coyuntura de fines de la dictadura militar que vivió el país entre los años 1973 y 1984, y los comienzos de la vida democrática para alcanzar los objetivos propuestos.

Este programa permitió potenciar la investigación científica en Uruguay, a través de la institucionalización de un sistema de posgrados científicos en ciencias básicas (sin perjuicio de meritorios antecedentes en ese campo en algunas facultades de la Universidad de la República). 
A diez años de la creación del PEDECIBA, en el año 1996, realizamos un estudio en el que buscábamos reconstruir el "período de iniciación" de este programa y que constituyó la primera aproximación sistemática a la fermental experiencia de este (Chiancone, 1996).

Treinta y tres años después de la fundación del programa y en un país que ha experimentado diversas transformaciones de la institucionalidad de la ciencia y la tecnología, creímos interesante revisitarlo. En esta oportunidad buscamos identificar algunas formas de estructuración y articulación del programa en el actual contexto institucional nacional.

El trabajo se basa en datos de fuentes secundarias: artículos científicos y de divulgación, documentos administrativos y legales, informes de evaluación del programa y notas de prensa.

\section{Flexibilidad de las organizaciones}

El concepto de flexibilidad ha sido definido de maneras muy diversas en distintos ámbitos. La variedad de tipos de flexibilidad en el contexto organizacional es entendida de diferente manera. En este sentido, los aportes sobre flexibilidad en las organizaciones han versado sobre flexibilidad e incertidumbre, flexibilidad estratégica y organizacional (Ansoff, 1975), organizaciones flexibles (Ackoff, 1977), empresas flexibles, así como sobre flexibilidad en las decisiones, entre otras varias (Sushil, 2016). Pese a los desarrollos de diversos conceptos, definiciones y modelos en el área de administración de los sistemas flexibles, la discusión teórica e intentos de creación de una teoría, han sido interpretadas como insuficientes por algunos autores. Ante esa falta de una teoría clara y comprensiva intentos como el de Sushil (2016) buscan proponer una teoría de la gestión de los sistemas flexibles.

Kickert (1985) se refería a la flexibilidad como la "palabra mágica" para resolver los problemas industriales y gubernamentales, y destacaba que desde las ciencias de la organización y la administración pública se hacían llamados al aumento de este rasgo en los sistemas organizacionales.

En el contexto de la administración pública y la definición de políticas, Kickert (1985), planteaba que la flexibilidad se refiere a la velocidad y el grado de cambio en los instrumentos para hacer frente a las contingencias medioambientales (Sushil, 2016). Por otro lado, que una organización sea más flexible implica que tiene una mayor capacidad de cambiar y ser cambiada. Si bien la flexibilidad no es una garantía de la adaptabilidad, es un requisito fundamental para ella (Ackoff, 1977).

Con estas referencias conceptuales, nos referiremos en este trabajo a uno de los rasgos más destacables del PEDECIBA, como la flexibilidad de la organización. Esta podría facilitar 
la adaptabilidad del programa, frente a cambios científicos a nivel global, e institucionales y de políticas a nivel local.

\section{Organización del Programa}

La condición institucional del programa es particularmente original, no sólo por la integración mixta entre organismos estatales, uno académico-UDELAR-, otro administrativoMEC- y una fuerte presencia de los actores, sino también porque posee una importante autonomía de funcionamiento. El convenio interadministrativo celebrado en 1986 entre UDELAR y el MEC establecía que las instituciones participantes del PEDECIBA no perdían identidad ni autonomía, sino que coordinarían y aunarían sus esfuerzos en la prosecución de un conjunto de fines específicos (PEDECIBA, 1986).|

Tres misiones integradas por investigadores extranjeros realizaron en 1989, 1996 y 2001, por iniciativa de las autoridades del programa, la evaluación del PEDECIBA. En el informe de la primera misión se destacaba que la preparación y el lanzamiento del programa habían exigido la discusión y regulación de aspectos muy complejos tanto desde el punto de vista práctico como jurídico. Se señalaba también que el PEDECIBA se apoyaba en cuatros puntos vinculados a los rasgos específicos del Uruguay y a las particulares condiciones del momento en el que fue concebido (MEC/ UDELAR, 1998):

i) el sentido de identidad nacional de la comunidad científica uruguaya;

ii) la amplia base de apoyo;

iii) la cooperación internacional; $\mathrm{y}$

iv) la implementación por los mismos científicos: La autogestión permitía la optimización del uso de recursos, por un lado, y por otro facilitaba la toma de decisiones, además de agilizar las distintas actividades. Una pequeña estructura administrativa, integrada por secretarias y bibliotecólogas de las áreas, y la existencia de muy pocos cargos (solamente los de Director, Secretaria del Director y personal auxiliar de dirección), llevaban a que las retribuciones del personal representaran solamente un 6\% del presupuesto del programa (este porcentaje ascendía al 8\% en 1996, como fuera señalado por la segunda misión).

En última instancia, han sido los propios investigadores quienes han sustentado el programa, incorporándose honorariamente al mismo, en el marco de las categorizaciones y regla- 
mentos aplicables, así como de regulares evaluaciones externas de los investigadores, coordinadas por las diferentes áreas. Esto ha evitado, además, subordinaciones de la política científica a posibles orientaciones de los organismos financiadores.

El programa ha operado como una instancia muy flexible y operativa a la vez, de organización de la investigación básica y de apoyo a otras unidades y departamentos científicos universitarios, con los cuales coopera financiando compras de equipos, materiales, bibliografía, participación en congresos y becas de estudiantes de posgrado.

La segunda y tercera misiones de evaluación subrayaban la independencia y agilidad del sistema en su gestión financiera, gracias a la colaboración de UNESCO y PNUD, que contrastaba con las frecuentes atrasos y lentitud en ese tipo de institución. Recomendaban mantener el mismo sistema administrativo y evitar la burocratización.

Autoridades del programa reconocían que la gran diferencia que existía antes entre el PEDECIBA y otras instituciones (como por ejemplo en materia de compras y apoyo administrativo), había disminuido en la actualidad. Esto era atribuido a una mejor comprensión de las necesidades de la ciencia y los investigadores de parte de la estructura administrativa de UDELAR y del estado. Identificaban en esas transformaciones, el rol pionero o transformador del programa (González, 2018).

\section{Las áreas}

El PEDECIBA estuvo en sus inicios integrado por cinco disciplinas fundamentales, a las que se denominó áreas: biología, física, informática, matemática y química.

Dichas disciplinas diferían marcadamente en términos de su madurez, grado de consolidación y masa crítica desarrollada en el período previo. Así, contrastando con la biología o la química -en cuya facultad homónima existió un doctorado, desde 1929, la informática era apenas una ciencia en desarrollo, que necesitaba aun consolidar sus núcleos de practicantes. A su vez, no existían en el país doctores en física. La matemática por su parte poseía líderes de la investigación bien conocidos en el mundo académico internacional, pero debía volver a echar raíces en el país, luego de su trasplante a otros medios y centros de investigación en el exterior.

Más tarde, en el año 2009 se creó una nueva área, geociencias, sumando así seis el total de áreas del programa. Un año después se puso en marcha un programa de maestría y en 2014 fue aprobado el doctorado de geociencias. 
Reiteradas recomendaciones de los evaluadores externos del programa sobre la necesidad de incentivar la formación y el trabajo interdisciplinario han sido integradas en los documentos oficiales del PEDECIBA como metas a alcanzar y se concretan en un conjunto de acciones. Como ejemplo de eso, cabe señalar la organización en 2009 de la maestría en bioinformática.

Esta maestría es un programa de posgrado del PEDECIBA y la UDELAR, con la participación del Instituto de Investigaciones Biológicas Clemente Estable (IIBCE) y del Instituto Pasteur de Montevideo. Cuenta además de los fondos centrales del programa con el apoyo financiero de la Agencia Nacional de Investigación e Innovación (ANII) obtenidos en el llamado a "Desarrollo de posgrados nacionales". La primera generación de quince estudiantes comenzó sus estudios de maestría en abril de 2009.

Por otro lado, ha sido elaborada una propuesta de maestría en nanociencias y ciencia de materiales (Universidad de la República, 2017).

En octubre de 2012, la "Semana de la Interdisciplina" buscaba impulsar la integración de distintas disciplinas a través de un conjunto de actividades (minicursos, talleres, conferencias, mesas redondas, entre otros) y presentación de pósters). Las actividades estaban orientadas a estudiantes e investigadores del programa, así como a otros académicos, profesionales y distintos actores del mundo productivo ( PEDECIBA, $\mathrm{s} / \mathrm{f} \mathrm{c}$ ).

Como lo ponía de manifiesto Ares Pons (2004) el PEDECIBA no posee personería jurídica propia y no administra una "carrera de investigador". Su conducción está muy próxima al cogobierno universitario, en la medida en que la Comisión Directiva, integrada por doce miembros está conformada por:

a) El Director y Subdirector Académicos.

b) delegados de la Universidad de la República y del MEC (tres y tres). Uno de los delegados del MEC es un investigador del Instituto de Investigaciones Biológicas Clemente (IIBCE).

b) un delegado de los investigadores y otro de los estudiantes de posgrado, y

c) dos delegados por el conjunto de los consejos de área, estos últimos electos por los "órdenes" (investigadores y estudiantes de posgrado).

Merece especial atención el criterio de que fueran dos delegados como representantes de las áreas y no uno por cada una de ellas. La necesidad de llegar a acuerdos sobre los dos delegados, o sobre decisiones a tomar en conjunto, obliga a establecer relaciones inter-áreas y a tomar decisiones que trascienden los particulares intereses y dinámicas de las diferentes disciplinas (Ares Pons, 2004). 
La actividad interna de las áreas está coordinada por los Consejos Científicos de Área. Estos son responsables de la regulación de las actividades de investigación y de formación de recursos humanos en su área. Cada consejo está integrado por i) cinco miembros elegidos entre los investigadores del área; ii) un delegado de los estudiantes (PEDECIBA, s/f b).

Por otro lado, considerando la doble pertenencia de los investigadores a dos culturas diferentes (Shils, 1991; Vessuri, 1996 b), una "universitaria" perfilada por la dinámica de la institución a la que pertenecen y sus conflictos, y otra cultura "internacional" de trabajo científico y evaluación del mismo, con la que están más familiarizados quienes estuvieron en el exterior, podría pensarse que la estructura del programa podría estar favoreciendo pautas asociadas a la cultura "internacional”. La presentación del área de Informática reconocía tales logros del programa:

“...creemos que el punto clave que ha hecho al PEDECIBA tan positivo fue la organización de la comunidad científica en torno a políticas, normas y pautas exigentes y, al mismo tiempo, adecuadas a nuestra realidad. La naciente área de Informática pudo crecer rápidamente debido a que poseía un importante grupo de estudiantes atraídos por la actividad académica y a que pudo recibir a través del PEDECIBA la experiencia y orientación del resto de la comunidad científica en un ambiente estimulante y constructivo" (PEDECIBA, $\mathrm{s} / \mathrm{f}$ ).

\section{$\underline{\text { Un modelo a imitar }}$}

El PEDECIBA ha sido y es percibido en los ámbitos académicos uruguayos como un modelo exitoso a emular. En ese sentido, en el año 2011 se firmó un convenio para la creación de un Programa de Desarrollo de Ciencias y Tecnologías Agropecuarias y Agroindustriales (PEDEAGRIND), con la coordinación interinstitucional del MEC, UDELAR, el Instituto Nacional de Investigaciones Agropecuarias (INIA) y el Laboratorio Tecnológico del Uruguay (LATU), pero el programa nunca llegó a concretarse. También existen en el país, dos proyectos de ley no aprobados relativos a la creación de un -Programa de Desarrollo de las Ciencias Sociales (PEDECISO) y de un Programa de Desarrollo Tecnológico (PEDETEC).

Se habría intentado además replicar el modelo del programa en otros países de Sudamérica, como por ejemplo en Paraguay (La Diaria, 2019)

\section{Cambios en el contexto institucional nacional de CTI}

En la transición hacia la democracia, desde mediados de los ochenta y a lo largo de los noventa, los gobiernos uruguayos implementaron políticas que enfatizaron el desarrollo tecnológico- pero sin formular una estructura general. Con la asunción del nuevo gobierno en el 
2004, tuvo lugar un cambio relativo en las orientaciones políticas y sociales globales, a la vez que un intento de reconstruir la institucionalidad de la ciencia y la tecnología.

En el año 2005 como parte de un proceso de rediseño institucional del sistema nacional de ciencia, tecnología e innovación (CTI) de Uruguay, se creó la Agencia Nacional de Investigación e Innovación (ANII) (Ley 17.930 de 2005), en un rol de ente ejecutor de las políticas, y de asesoramiento al Poder Ejecutivo, en materia de instrumentos y programas para la promoción y fortalecimiento de CTI. Sus cometidos y competencias fueron establecidos por la Ley N. ${ }^{\circ}$ 18.084 de 2006. En el ámbito de la ANII, en el año 2007, se creó el Sistema Nacional de Investigadores (SNI), Fue fundado para fortalecer, expandir y consolidar la comunidad científica nacional; además de realizar las actividades de categorización y evaluación periódica de todos los investigadores, y el establecimiento de un sistema de incentivos económicos.

Ares Pons (2004) subrayaba asimismo las valoraciones positivas recogidas en sus evaluaciones externas y su constitución, de hecho, en un referente nacional e internacional, con funciones de "una verdadera Academia Nacional de Ciencia Básica", que recién sería creada por la Ley 18.582, aprobada el 2 de Setiembre del 2009.

A medida que se fue desarrollando el proceso de diversificación del sistema de investigación y desarrollo (I+D) uruguayo comenzaron a coexistir nuevos actores y el PEDECIBA perdió centralidad. Sin embargo, pese a haber dejado de ser el protagonista principal con múltiples roles, ahora asumidos por otras organizaciones, el programa persiste y ha sido un modelo para varias iniciativas más jóvenes.

Por otro lado, en el país fueron creadas nuevas instituciones de educación superior (en el ámbito privado desde el año 1984) e institutos de investigación científica, que se integraron a la I+D local.

Hoy el programa cuenta con 900 investigadores honorarios y 1.000 estudiantes de posgrado (La Diaria, 2019)

El reglamento del PEDECIBA en su versión del año 2018 establece que,

Un Investigador Activo del PEDECIBA es aquel que mantiene una vinculación laboral con una institución pública o privada en la que se realiza investigación en el país, tiene producción científica reciente y participa en la formación de investigadores. Cada Investigador será asignado en uno de los grados que se describen a continuación [...]

Es así como un investigador puede pertenecer al PEDECIBA e integrar la red de colaboración construida en torno a los objetivos del programa, a la vez que tener un cargo en una 
institución académica. La pertenencia a la red les permite disponer de recursos de manera ágil, con un mínimo de trámites burocráticos y con un manejo transparente de fondos públicos (Lessa y Torre, 2011). Los investigadores del programa integran hoy el cuerpo académico de un espectro más amplio de organizaciones, tales como el Instituto Nacional de Investigaciones Agropecuarios (INIA), el Instituto Pasteur y la Universidad ORT.

En el ámbito de un creciente desarrollo y complejidad de las ciencias a nivel global, y de cambios institucionales y políticas de CTI locales, PEDECIBA es una red de investigadores administrada por los propios investigadores, a la que se han integrado nuevos campos interdisciplinarios y se ha ampliado el número de instituciones uruguayas donde trabajan los científicos del programa.

\section{Investigadoras e investigadores del PEDECIBA}

Los "investigadores activos" del programa son clasificados en uno de los tres grados posibles: Grados 3, 4 o 5, según sus méritos en su nivel de formación, producción científica, formación de recursos humanos, trayectoria en su disciplina de trabajo, y reconocimiento nacional e internacional (PEDECIBA, 2018).

En relación con la distribución de investigadores activos del PEDECIBA por sexo, Bielli y Chiancone (2016), señalaban que la comparación de los datos de los años 2005, 2009 y 2014, indicaba que los hombres constituían a mayoría de los investigadores activos, pero que su proporción tendía a disminuir con el transcurso del tiempo, como se indica en la Tabla N. ${ }^{\circ} 1$.

Tabla No. 1: Distribución de investigadores activos del PEDECIBA por sexo. Años 2005, 2009 y 2014.

\begin{tabular}{|c|c|c|c|}
\hline & Hombres & Mujeres & Total \\
\hline 2005 & $299(65 \%)$ & $161(35 \%)$ & $430(100 \%)$ \\
\hline 2009 & $362(59,7 \%)$ & $244(40,3 \%)$ & $606(100 \%)$ \\
\hline 2014 & $382(54,6 \%)$ & $318(45,4 \%)$ & $700(100 \%)$ \\
\hline
\end{tabular}

Fuente: Bielli y Chiancone (2016)

Según planteaban las autoras, el aumento de la participación femenina, se corresponde con el incremento de participación de las mujeres en todas las áreas con excepción de informática, y por el ingreso sostenido de mujeres a las disciplinas tradicionalmente feminizadas como la química y la biología, así como también por la disminución de la brecha entre hombres 
y mujeres en Matemáticas. En contraste con el aumento de la participación de mujeres en el conjunto de investigadores en el período analizado, destacaban una segregación vertical: en los grados más altos de la categorización del programa era menor la presencia femenina, como puede verse en la Tabla N. ${ }^{\circ} 2$.

Tabla $N^{\circ}$ 2. Distribución por sexo y grado de investigadores (personas físicas) activos PEDECIBA. Años 2005, 2009 y 2014.

\begin{tabular}{|c|c|c|c|c|c|c|c|c|c|c|}
\hline & \multicolumn{5}{|c|}{$\frac{\text { GRADO } 3}{\text { Sexo }}$} & \multicolumn{5}{|c|}{$\frac{\text { GRADO } 4}{\text { Sexo }}$} \\
\hline AÑOS & $F$ & $\%$ & M & $\%$ & TOTAL F+M & $F$ & $\%$ & M & $\%$ & TOTAL F+M \\
\hline 2005 & 106 & 49.5 & 108 & 50.5 & 214 & 40 & 30.3 & 92 & 69.7 & 132 \\
\hline 2009 & 179 & 53.9 & 153 & 46.1 & 332 & 49 & 32.5 & 102 & 67.5 & 151 \\
\hline \multirow[t]{2}{*}{2014} & 237 & 56.6 & 182 & 43.4 & 419 & 66 & 38.4 & 106 & 61.6 & 172 \\
\hline & \multicolumn{5}{|c|}{$\frac{\text { GRADO } 5}{\text { Sexo }}$} & \multicolumn{3}{|c|}{$\begin{array}{c}\text { TOTAL PEDECIBA } \\
\text { Sexo }\end{array}$} & & $\begin{array}{c}\text { Total } \\
\text { PEDECIBA }\end{array}$ \\
\hline ANNOS & F & $\% \mathrm{~F}$ & $M$ & $\% \mathrm{M}$ & TOTAL F+M & F & $\% \mathrm{~F}$ & M & $\% \mathrm{M}$ & $\mathrm{F}+\mathrm{M}$ \\
\hline 2005 & 15 & 13.2 & 99 & 86.8 & 114 & 161 & 35.0 & 299 & 65.0 & 460 \\
\hline 2009 & 16 & 13.0 & 107 & 87.0 & 123 & 244 & 40.3 & 362 & 59.7 & 606 \\
\hline 2014 & 15 & 13.8 & 94 & 86.2 & 109 & 318 & 45.4 & 382 & 54.6 & 700 \\
\hline
\end{tabular}

Fuente: Elaboración propia en base a Bielli y Chiancone, 2016

Esta situación de segregación vertical se da también en las áreas donde participan mayoritariamente hombres, como física y matemática y la más nueva, geociencias, donde se presentan situaciones tales como la inexistencia de mujeres grado 5 , en algunos de los años analizados. En informática el avance de las mujeres hacia la categoría más alta es cero en todo el período. Las autoras concluían que aparentemente, en el conjunto y con diferentes énfasis, las mujeres encontrarían obstáculos para avanzar en las categorías académicas en todas las áreas del programa (Bielli y Chiancone, 2016: 95).

La situación descrita es coherente con lo que sucede en los ámbitos de actividades de CTI en Uruguay y la región. A pesar del aumento de la participación de mujeres en las instituciones educación superior y CTI, pueden observarse todavía fenómenos de segregación horizontal (mayor presencia de hombres en relación con mujeres en ciertas disciplinas), y de segregación vertical (menor presencia femenina en los niveles de mayor jerarquía) (Bielli, Buti y Viscardi, 2001a, 2001b; Bernheim, 2015; Bielli y Chiancone, 2016)

Existen diversas interpretaciones de las situaciones de esta disparidad en los distintos espacios de CTI. Por un lado, se plantea que la cuestión de género podría ubicarse hoy en el mercado laboral, dado que a diferencia de lo que acontecía décadas atrás en los espacios de formación, la presencia de las mujeres llega a veces a superar la de los hombres. Sin embargo, otras perspectivas, destacan la vigencia y la relevancia de la cuestión de género; a pesar de la participación femenina en términos cuantitativos, persisten condiciones para los hombres, 
que favorecen el desarrollo académico ventajoso en relación con el de las mujeres (Vessuri y Canino, 2007)

En setiembre de 2019, como parte de un proyecto presentado por un consorcio de seis países a la convocatoria Horizonte 2020 SWAFS, se desarrolló el taller "Hacia la implementación de planes de equidad de género en organizaciones de I+D en Uruguay: PEDECIBA". Dicha propuesta tiene como objetivo fortalecer los procesos de implementación de planes de equidad de género en organizaciones de investigación y organismos que tienen programas de apoyo a la investigación académica de diferentes regiones de Europa y América Latina.

El taller fue realizado en el Área Química del PEDECIBA y se pretendía realizar ejercicios piloto de discusión y diseño, con la participación de investigadores e investigadoras de esa área.

\section{Principales desafíos}

Los desafíos a los que se enfrenta el programa 33 años después, incluyen aspectos como el financiamiento, la retención de investigadores, la evaluación de los cursos y las áreas, entre otros que implican las demandas de los diversos actores del PEDECIBA.

Nos referiremos solamente los dos primeros, por ser quizás los más críticos, y por estar directamente vinculados con la viabilidad del programa y su impacto en la sociedad uruguaya.

\section{El financiamiento:}

El PEDECIBA logró institucionalizarse más allá del momento favorable de la transición democrática, pero debió hacer frente a la escasez de los recursos y sostener su actividad y expandir sus líneas de investigación. El programa ha sido sometido a evaluaciones externas desde el año 1989 y varios indicadores reflejan su significación y su impacto. La principal incertidumbre del programa parecería ser la continuidad de los ingresos necesarios para el desarrollo de las actividades del programa.

Entre 1993 y 1997 el CONICYT colaboró en la administración de fondos del préstamo CONICYT-BID para ciencia y tecnología. La ley de presupuesto nacional de 1995 estableció al PEDECIBA como programa permanente (Ley 16736, Arts. 386 y 387). Sin embargo, los recortes presupuestales experimentados en el país a partir de la crisis regional del 2002 lo afectaron críticamente. Como lo expresara la Comisión Directiva en una carta a los integrantes del Equipo Operativo del Gabinete de Innovación: 
La creación del PEDECIBA y su desempeño en estas dos décadas, produjeron la falsa sensación de que el país habia encontrado una fórmula casi infalible para desarrollar una infraestructura científica básica nacional suficiente con un costo mínimo (PEDECIBA, 2008: 1).

Como consecuencia de esto, los programas BID CONICYT, y el PDT, concentraron sus esfuerzos fundamentalmente en promover la investigación tecnológica en áreas temáticas definidas como prioritarias y en la innovación. Fueron escasos los recursos destinados a financiar investigación fundamental y con muchas limitaciones para su ejecución.

La escasez de recursos habría afectado especialmente a las áreas más grandes, debido a los criterios establecidos inicialmente para la distribución de los fondos, que habrían beneficiado a las áreas más pequeñas y de escaso desarrollo. En ese sentido, la Comisión de Presupuesto de biología (2006) señalaba la grave situación en los diferentes programas del área:

a) las becas fueron restringidas a los "estudiantes excepcionales"; como consecuencia de ello más de la mitad de los aspirantes no fueron apoyados. Por otro lado, las becas fueron reducidas en su duración (menor que las otras áreas), y en los montos; mayoritariamente no representaba una beca total, sino que se limitaba a completar otros ingresos - por ejemplo, salarios docentes universitarios;

b) el sistema de pasantías de estudiantes en el extranjero fue cancelado;

c)los apoyos excepcionales al presupuesto de cursos fueron recortados y consecuentemente no se ha financiado la venida de profesores visitantes.

A partir de su creación la Agencia Nacional de Investigación e Innovación (ANII) asumió la viabilidad de becas de posgrado en el PEDECIBA. Esto dio un poco de margen al presupuesto propio de programa (unos 7,5 millones de pesos). Así, las becas llegaron en 2008 a 120.

En el nuevo acuerdo MEC-UDELAR de fecha 17 de abril 2008, el Gobierno de la República a través del MEC y UDELAR conviene en "aunar esfuerzos para continuar coordinando sus actividades para el desarrollo y el mantenimiento de la investigación en ciencias básicas, adaptando al PEDECIBA a la nueva realidad del país y de la región, otorgándole un carácter de programa permanente". Además, la UDELAR y el Gobierno uruguayo se comprometen a "intentar asumir gastos permanentes de la investigación científica (biblioteca, apoyo técnico y administrativo, etc.) en sus presupuestos, liberando así recursos que el PEDECIBA podrá aplicar para ayudar a la implementación de sus nuevos objetivos" (MEC/ UDELAR, 2008).

\section{La retención de investigadores}

Entre los objetivos del programa se encuentra el promover "el retorno al país de investigadores en ciencias básicas radicados en el exterior, procurando crear condiciones adecuadas 
para que mantengan su nivel de producción científica" y que contribuyan a que se cumplo los restantes objetivos del programa (PEDECIBA, 1986).

Su aporte en términos de estímulo a la migración de retorno de los científicos fue importante en algunos casos, ello implicó la reimplantación de comunidades disciplinarias enteras, como en el caso de los matemáticos (Chiancone, 1997).

"Uruguay Retiene" es una iniciativa en la que el PEDECIBA convoca a interesados en realizar bajo contrato por un año, actividades de investigación en instituciones que se dedican a ese rubro (contrato renovable por un año más sujeto a disponibilidad de presupuesto). Los aspirantes deberán haber obtenido el título de doctorado en los últimos tres años. A través de estos contratos se busca apoyar a investigadores a radicarse y consolidarse en el país y evitar la emigración de personal altamente calificado. En 2017 fueron contratados quince recientes doctores, jóvenes investigadores científicos.

\section{Conclusiones}

El PEDECIBA exhibió claramente en estos treinta y tres años su viabilidad, su éxito en la consolidación de las disciplinas científicas en el país, su eficaz construcción de un sistema de posgrados científicos en sentido estricto. Su original estructura institucional lo preservó de su rutinización, de la absorción por las dinámicas internas y burocráticas de las instituciones que lo constituyen y sostienen, a la vez que evitó entrar en competencia con otras instituciones científicas, sino que por el contrario se amplió el conjunto de instituciones donde trabajan sus investigadores generando sinergias.

Más allá del grado en que la nueva institucionalidad afecte al sistema nacional de CTI, el nuevo escenario del Uruguay y de la región, encuentra un PEDECIBA sumamente consolidado y visible.

En el desarrollo del PEDECIBA puede reconocerse un rol clave jugado por las disciplinas básicas fundacionales, cuya institucionalización nos permite reconocer dos tendencias sincrónicas pero diversas: por un lado, la fortificación de la masa crítica local, a través de mecanismos de afiliación institucional, en simultáneo con la profundización de las relaciones de cooperación y de internacionalización aportados por los líderes y por otros científicos.

En un contexto de recreación institucional de las políticas de ciencia y tecnología, no exentas de críticas y discusiones entre segmentos de investigadores ligados a los laboratorios y los gestores gubernamentales de la política de I+D, el PEDECIBA, parece excluido como 
objeto de debate. Constituye una política pública exitosa, que gradualmente ha adquirido un tinte de política de estado.

Algunos desafíos para el futuro del programa están asociados a dar respuesta a un conjunto de demandas y problemas. Algunas de las fuentes de éstos, son: a)entre los externos, los cambios en la estructura productiva nacional, y la orientación de la política científico-tecnológica que empieza a subrayar con fuerza la relevancia de la innovación tecnológica asociada a los procesos de generación de conocimiento; la insuficiente inversión pública y privada en actividades de I+D; los riesgos del brain drain de las capacidades existentes;

b) entre las internas: la equidad de género en el ingreso, permanencia y ascenso en las distintas áreas y en los grados de categorización; la respuesta a los pedidos de los estudiantes e investigadores, entre otros actores implicados.

Evaluado como caso "ejemplar" que recientemente cumplió 33 años de vida, el PEDECIBA estuvo desde sus orígenes fuertemente anclado a las necesidades nacionales y a una gran vocación internacional. Es una red de investigadores administrada por los propios científicos. Se han integrado nuevos campos interdisciplinarios y ha aumentado la cantidad de instituciones uruguayas donde trabajan los investigadores del programa. Con algunos desafíos asociados a los cambios científicos a nivel global, e institucionales y de políticas locales, el programa mantiene su importancia en la formación avanzada de recursos humanos y en la coordinación y creación de un espacio de reunión de investigadores.

\section{Referencias citadas}

Ackoff, R.L. (1977) “Towards flexible organizations: A multidimensional design”. Omega, Vol. 5, $\mathrm{N}^{\circ}$ 6, pp. 649-662. ISSN: 0305-0483.

Ansoff, H. I. (1975). Managing strategic surprise by response to weak signals. California Management Review, 8(2), pp. 21-33.

Aráoz, J., Baran, E., Cardinali, D., Corach, G., Grigera. J. R., Orszag, M. (2001). Informe de la Misión de Evaluación del Programa para el Desarrollo de las Ciencias Básicas. (Noviembre de 2001). Informe Global. (Mimeo).

Ares Pons, J. (2009). "El Programa para el Desarrollo de las Ciencias Básicas (PEDECIBA)". Versión con Addenda al trabajo de 2004 del mismo nombre. Recuperado de https://sites.google.com/site/jorgearespons/home/universidad-y-pedeciba. 
Bernheim, R. (2015). Informe de género año 2015. Montevideo: ANII. Unidad de Evaluación y Monitoreo.

Bielli, A., Buti, A. y Viscardi, N. (2001a). Mujeres investigadoras uruguayas: ¿participación real o incierta? Documento de Trabajo No. 9, CSIC. Recuperado de http://www.csic.edu.uy/renderPage/index/pageId/433

Bielli, A., Buti, A. y Viscardi, N. (2001b). "Participación de mujeres en investigación científica de nivel universitario en Uruguay" en Las mujeres en el sistema de Ciencia y Tecnología. Estudios de casos, E. Pérez Sedeño (Ed.). Madrid: OEI.

Bielli A. y Chiancone A. (2016) "Estudio sobre Ciencia, Tecnología y Género - Informe Uruguay” Red Iberoamericana de Ciencia Tecnología y Género (RICTYG).

Casas, R. (2001). "El enfoque de redes y flujos de conocimiento en el análisis de las relaciones entre ciencia, tecnología y sociedad" en KAIROS - Año 5 Nro. 8, 2do. Semestre 2001 ISSN 1514-9331.

Chiancone, A. (1996). La creación de políticas públicas en un contexto de transición política: El caso del PEDECIBA en Uruguay. Buenos Aires: FLACSO-DAAD.

Chiancone, A. (1997). "Los matemáticos uruguayos: una historia de migraciones". En: Redes. Vol. IV, N. ${ }^{\circ}$ 10. Buenos Aires: Universidad de Quilmes.

Chiancone, A. (2018). “Ciencia, Tecnología e Innovación” en OPP/ MIDES “Reporte Uruguay 2017". Proyecto URU/07/002-PNUD. Recuperado de http://200.40.96.180/images/ReporteUruguay2017.pdf

González, D. (2018) “PEDECIBA 2.0 Desafíos y horizontes actuales de PEDECIBA". Recuperado de http://www.pedeciba.edu.uy/election-director/Propuesta_de_trabajo_David_Gonzalez.pdf

Kickert, W. J. M. (1985). The magic word flexibility. International Studies of Management and Organization, XIV(4), pp. 6-31.

La Diaria (2019) "El Programa de Desarrollo de las Ciencias Básicas: una usina de ideas“. Publicado el 29 de junio de 2019. Recuperado de https://ciencia.ladiaria.com.uy/articulo/2019/6/el-programa-de-desarrollo-de-las-ciencias-basicas-una-usina-de-ideas/

Lesa E. y Garat, B. (2011). "PEDECIBA. Respondiendo a un desafío que perdura. Uruguay Ciencia No. 13 - Octubre 2011 
MEC/UDELAR .(1986). "Convenio creación del PEDECIBA (1986)". Recuperado de http://www.pedeciba.edu.uy/docspd/acuerd86.htm

MEC/UDELAR .(1998). "Informe final del Proyecto URU/84/002 PEDECIBA”. Recuperado de http://www.pedeciba.edu.uy/docspd/inffin98.htm

MEC/UDELAR .(2008). “Convenio para el desarrollo de las Ciencias Básicas entre el Ministerio de Educación y Cultura y la Universidad de la República". Recuperado de http://www.pedeciba.edu.uy/docspd/AcuerdoAbril08.pdf

PEDECIBA (s/f a). “Área de Informática. Antecedentes y evolución histórica”. Recuperado de http://www.pedeciba.edu.uy/docspd/infinf.htm

PEDECIBA (s/f b). "Sobre PEDECIBA". Recuperado de http://www.pedeciba.edu.uy/sobrepedeciba.php

PEDECIBA .(s/f c). "Semana de la Interdisciplina 2012". Recuperado de http://www.pedeciba.edu.uy/ei2012/indice.html

PEDECIBA (1998) “Informe final del Proyecto URU/84/002 PEDECIBA". Recuperado de

http://www.pedeciba.edu.uy/docspd/inffin98.htm

PEDECIBA (2006). Carta a los Miembros del Equipo Operativo del Gabinete Ministerial de la Innovación. 26 de agosto 2006. Recuperado de http://www.pedeciba.edu.uy/AequipOper.pdf

PEDECIBA (2008 a). "Informe de Actividades 2008". Recuperado de http://www.pedeciba.edu.uy/docspd/INFORMEACTIVIDADES_CEN_08.pdf

PEDECIBA (2008 b). "Convenio para el Desarrollo de las Ciencias Básicas entre el Ministerio de Educación y Cultura y la Universidad de la República". Recuperado de http://www.pedeciba.edu.uy/docspd/AcuerdoAbril08.pdf

PEDECIBA (2013) Reglamento del personal académico del PEDECIBA, 2013”. Recuperado de http://www.pedeciba.edu.uy/docspd/ReglamentoPersonalAcademico27_de_\%20junio_de_2013.pdf

PEDECIBA (2016) "Uruguay retiene". Recuperado de http://www.pedeciba.edu.uy/docspd/URUGUAY\%20RETIENE_final.pdf

PEDECIBA (2018) "Categorías del personal académico del PEDECIBA". Recuperado de http://www.pedeciba.edu.uy/docspd/ReglamentoPersonalAcademico_version2018.pdf 
Shils, E. (1991). Reflections on tradition. Center and Periphery and the Universal Validity of Science: The Significance of the life of S. Ramanujan" en Minerva Vol.XXIX, No 14, pp. 393-419.

Sushil. (2016). "Theory of Flexible Systems Management" en Sushil, J. Connell y Burgess (eds.) Flexible Work Organizations: The Challenges of Capacity Building in Asia Springer: New Delhi, pp. 3-20.

Universidad de la República. (2012). Datos básicos de I Censo de Estudiantes Universitarios de Posgrado año 2012. Montevideo: UDELAR. Recuperado de http://planeamiento.udelar.edu.uy/files/2013/09/triptico_posgrado_pdf.pdf

Universidad de la República. (2013). Memoria 2013. Montevideo: UDELAR. Recuperado de http://www.universidad.edu.uy/renderPage/index/pageId/710\#heading_2413

Universidad de la República (2016)، “30 años de PEDECIBA. La senda está trazada” Publicado el domingo 23 de octubre de 2016. Recuperado de http://universidad.edu.uy/prensa/renderItem/itemId/39715

Universidad de la República. (2017). "PEDECIBA: 30 años empujando el muro del conocimiento" Publicado el martes 14 de marzo de 2017. Recuperado de http://www.universidad.edu.uy/prensa/renderItem/itemId/40160/refererPageId/12 